\title{
Modeling neuronal firing in epilepsy - fitting Hawkes processes to single-unit activity
}

\author{
György Perczel, Loránd Erőss, Dániel Fabó, László Gerencsér, Zsuzsanna Vágó
}

\begin{abstract}
Forecasting seizures based on information extracted from neuronal firing has a great potential in controlling closed-loop neurostimulators. For the description of neuronal firing patterns we use self-exiting point processes or Hawkes processes. In fitting them to simulated data, using a large variety of models, we consider both computability and reliability issues related to the maximum likelihood estimation (MLE) method. The models are classified via a single parameter related to stability regimes. The dependence of the accuracy of the individual parameter estimates on different regimes will be explored. We demonstrate the applicability of the MLE method to discriminate between different models with high confidence.
\end{abstract}

\section{Introduction}

\subsection{A brief introduction to epilepsy}

With a prevalence of $0,5-1 \%$ epilepsy is one of the most common neurological disorders. Its most characteristic features are recurrent seizures. Despite that a number of causes have already been identified, including genetic or cerebrovascular disorders, brain injury and infections, 6 out of 10 cases are categorized as idiopathic, i.e. the

Gyögy Perczel, Loránd Erőss, Dániel Fabó

National Institute of Clinical Neurosciences, Budapest, Hungary, and Faculty of Information Technology and Bionics, Pázmány Péter Catholic University, Budapest, Hungary

László Gerencsér

Institute for Computer Science and Control, Hungarian Academy of Sciences, Budapest, Hungary

Zsuzsanna Vágó

Faculty of Information Technology and Bionics, Pázmány Péter Catholic University, Budapest, Hungary

Contact: gerencser.laszlo@sztaki.mta.hu 
main cause is unknown. Numerous anti-epileptic drugs are available and in some cases even surgical options exist, such as excision of the epileptogenic focus or the implantation of a neurostimulator. However, for approximately $30 \%$ of the patients sufficient seizure control cannot be achieved, see [14].

The unexpectedness of the seizures has such an influence on the patients' wellbeing that reducing the frequency of seizures only moderately improves their quality of life. Even in the case of rare seizures, the patient's life is determined by the fear of a forthcoming one [14]. Thus, it is generally accepted, that a system capable of forecasting seizures would ameliorate the quality of life of patients. Furthermore, it is hypothesized that combining such a system with a neurostimulator or a drugdelivery pump the development of seizures could be avoided [4].

\subsection{Seizure prediction and closed-loop neurostimulators in epilepsy}

In order to forecast forthcoming seizures numerous methods have been proposed, see [4]. Most often these are based on the analysis of the electrical signals of the brain, such as the ones recorded on the scalp (electroencephalogram, EEG) or on the surface of the brain (electrocorticogram, ECoG). It is assumed that focal seizures starting from a distinct cortical area evolves as a cascade of events and thus can be theoretically predicted, though we are not aware of a specific biomarker preceding them [9]. Although some methods satisfy current statistical criteria (above chance), applicability in a clinical setting requires more rigorous standards [4].

We note that experimental and modeling studies carried out hitherto suggest that some seizures are inherently unpredictable. This is the case for primary generalized absence seizures that involve both cortical hemispheres from the very beginning of the onset of the seizure. A brain with this type of seizures is regarded as a bistable system that switches between its states stochastically [9].

To date, there is only one implantable closed-loop system available on the market, the Responsive Neurostimulation or RNS (NeuroPace, CA, USA). However, this device detects seizures, instead of predicting them, and delivers electrical stimulation to the area thought to be responsible for the seizure initiation. In contrast, the Seizure Advisory System (NeuroVista, WA, USA) aimed to achieve real seizure forecasting, but is not yet available on the market, [4].

The tools for seizure prediction mentioned above utilize the low-frequency components of brain electrical signals called local field potentials (LFP) ranging from approximately $0.1 \mathrm{~Hz}$ to $200-300 \mathrm{~Hz}$. As an alternative the appropriate signals can be filtered using a high pass filter with a cutoff frequency around $200-500 \mathrm{~Hz}$. The resulting time-series (multi-unit activity, MUA) will contain primarily the action potentials of neurons from the vicinity of the recording electrodes. With further processing, called spike-sorting, these APs can be assigned to individual units (neurons) based on their morphology. This provides us sequences of time-points indicating the APs of distinct units termed single-unit activities (SUAs) [13]. As there is evidence that epileptic activity is a result of the underlying pathological neuronal firing [10] 
the question arises if seizure prediction can be achieved based on the investigation of SUAs [15]. In this paper we provide a framework for the statistical analysis of SUAs using the theory of point processes, and give a summary of our extensive simulation-based investigations.

\section{Modeling single-unit activity via Hawkes processes}

The series of time points of the APs is modeled with a so called point process, [2]. Mathematically this is a strictly increasing sequence of random time points $0=T_{0}<$ $T_{1}<T_{2} \ldots$ with no accumulation point. For the sake of mathematical convenience we will also consider two-sided point processes $\left(T_{n}\right),-\infty<n<\infty$, for which the range of $T_{n}$-s is $(-\infty,+\infty)$. For an excellent introduction see [1].

An alternative description of a point process is given by its counting process defined for the one-sided point process as $N_{t}=\#\left\{n: 0<T_{n} \leq t\right\}$. More generally, we may define a counting measure for any interval $(a, b]$ by the equation $N(a, b]=$ $\#\left\{n: a<T_{n} \leq b\right\}$. The internal history or the past of a point process is defined as the $\sigma$-algebra

$$
\mathscr{F}_{t}=\sigma\{N(a, b]: a<b \leq t\} .
$$

The definition of counting measure and internal history works equally for one-sided and two-sided point processes. We define the integral of a random so-called predictable function $f_{t} \geq 0, t \geq 0$ with respect to $d N_{t}$. The heuristic meaning of predictability is that $f_{t}$ is the limit of left-continuous $\mathscr{F}_{t}$-adapted processes. Then set

$$
\int_{0}^{\infty} f_{t} d N_{t}=\sum_{n \geq 0} f\left(T_{n}\right)
$$

It can be shown that associated with $d N_{t}$ is a so-called (predictable) intensity process with the property that

$$
\mathrm{E}\left(\int_{0}^{\infty} f_{t} d N_{t}\right)=\mathrm{E}\left(\int_{0}^{\infty} f_{t} \lambda_{t} d t\right) .
$$

A prominent class of point processes in the field of neuroscience, emulating the firing pattern of a network of neurons interacting via APs in the brain, is the class of (multi-variate) mutually exiting point processes, or Hawkes processes, introduced in [8], see also [1], [2], [7] and [6].

A two-sided multivariate point process, $\left(T_{i, n}\right), i=1, \ldots, k$, is a Hawkes process, if its counting measures $N_{i}($.$) are shift invariant in time, with intensity functions$

$$
\lambda_{i, t}=\mu_{i}+\sum_{j=1}^{k} \int_{-\infty}^{t} g_{i j}(t-s) d N_{j, s}, \quad \mu_{i}>0, g_{i j}(u) \geq 0 .
$$


Here $\mu_{i}$ are the background intensities, and $g_{i j}(u)$ are non-negative impulse response functions (IRF). For the analysis of the firing pattern of a single unit we use a univariate (self-exciting) Hawkes process, implicitly defined by the feedback loop

$$
\lambda_{t}=\mu+\int_{-\infty}^{t} g(t-s) d N_{s}, \quad \mu>0, g(u) \geq 0 .
$$

Taking expectation on both side, and setting $\bar{\lambda}=\mathrm{E} \lambda_{t}$, we get the equation $\bar{\lambda}=$ $\mu+c \bar{\lambda}$, and the necessary (and sufficient, see [11]) condition for the existence of a Hawkes process satisfying (2):

$$
c=\int_{0}^{\infty} g(t) d t<1 .
$$

The rational behind the application of Hawkes processes in the analysis of SUA is that the burst-mode of neurons indicates a feedback-effect. The objective of the present study is to model individual neurons' firing pattern by fitting a univariate Hawkes process via the maximum-likelihood method, see [12], and to provide a summary of extensive experimental findings based on simulated data. In particular, we explore the typical configurations in the parameter space and establish confidence limits for discerning different regimes.

\section{Statistical fitting of Hawkes processes}

In order to fit Hawkes processes to real SUA data we consider a parametric class of Hawkes processes with

$$
g(u)=\sigma \cdot e^{a u}, u \geq 0, \quad \text { with } \quad \sigma>0, a<0,
$$

see [12]. In this case the stability criteria (3) becomes $-\sigma / a<1$, or equivalently, $\alpha:=a+\sigma<0$. Here $\alpha$ is called the stability margin. Let $\eta=(\mu, a, \sigma)$, and assume that our data are in fact generated by a Hawkes process defined above with true parameter $\eta^{*}$. To estimate this we take an arbitrary feasible parameter $\eta$, satisfying the conditions $a+\sigma<0<\mu$, and define an intensity function $\lambda_{t}(\eta)$ :

$$
\lambda_{t}(\eta)=\mu+\int_{0}^{t} g(t-s, \eta) d N_{s}=\mu+\int_{0}^{t} \sigma \cdot e^{a(t-s)} d N_{s} .
$$

The computation of the (conditional) log-likelihood function, under the condition that $d N_{t}=0$ for $t \leq 0$, is the mathematically substantiated heuristics that under minimal conditions a point process is locally a Poisson process, see $[3,12]$. Thus, the negative log-likelihood function on the interval $[0, T]$ is, modulo constants, 


$$
L_{T}(\eta)=\int_{0}^{T} \lambda_{t}(\eta) d t-\int_{0}^{T} \log \lambda_{t}(\eta) d N_{t} .
$$

For a rigorous foundation we refer to [12], and [3] for a more up to date reference.

The apparently cumbersome computation of the (predictable) intensity $\lambda_{t}(\eta)$ is actually quite simple for an exponential IRF. Namely, it follows directly from (4), by moving $\mu$ to the 1.h.s. and then differentiating w.r.t. $t$, that on the interval $T_{n-1}<$ $t \leq T_{n}$, where no event occurs, we have

$$
\lambda_{T_{n-1+}}=\lambda_{T_{n-1}}+\sigma, \quad \lambda_{t}(\eta)-\mu=e^{a\left(t-T_{n-1}\right)}\left(\lambda_{T_{n-1+}}(\eta)-\mu\right) .
$$

To bring the model closer to physiological reality we introduce an alternative parameterization using the stability margin and the average intensity as parameters, thus obtaining $\theta=(\alpha, \sigma, \bar{\lambda})$. As a measure of the precision of our estimators we use 95\% confidence-ellipsoids. We note that the Fisher information matrix, for a general parameteric class of Hawkes processes, is obtained from (5) as follows:

$$
I\left(\theta^{*}\right)=\lim _{T \rightarrow \infty} \frac{1}{T} \sum_{0<T_{n} \leq T} \frac{\lambda_{\theta T_{n}}\left(\theta^{*}\right) \cdot \lambda_{\theta T_{n}}^{T}\left(\theta^{*}\right)}{\lambda_{T_{n}}^{2}\left(\theta^{*}\right)},
$$

where the subscript ${ }_{\theta}$ denotes differentiation w.r.t. $\theta$, assuming the validity of an appropriate strong law of large numbers.

\section{Experimental results}

We implemented the above method in MATLAB and tested its performance. The accuracy of the MLE method was tested using simulated data generated by an improved version of the procedure presented in [12]. The length of an experiment is defined via the number of simulated events, which is in the range of 10.000 in our case. The accuracy of the estimators are characterized by confidence ellipsoids defined for level $95 \%$. The scope of experimental studies was focused on the sensitivity of the method w.r.t. changes in model dynamics, including changes in the orientation of the respective confidence ellipsoids.

On Figure 1 we present the confidence ellipsoids of two simulated SUAs, taken as benchmark examples. The processes were simulated with $N=10.000$ events with $\theta=(-2.1 ; 0.9 ; 1.0)$ and $\theta=(-0.6 ; 2.4 ; 1.0)$, denoted with green and red, respectively. In order to enhance our potential to discriminate between two models we can increase the number of observed events. We note that the volume of a confidence ellipsoid, denoted by $V_{C E}$, corresponding to a fixed model, based on $N$ events is proportional to $N^{-3 / 2}$. However, in a real-life situation when estimating the dynamics during the preictal period, the number of events associated with a stationary regime is limited due to changes in the dynamics close to the onset of a seizure. 


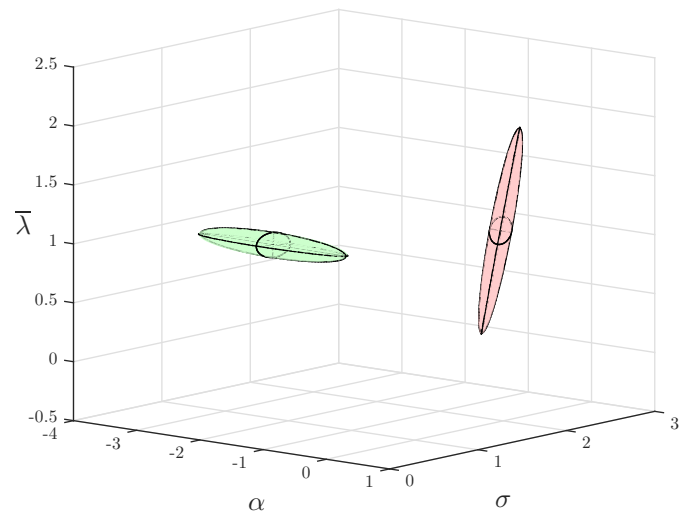

Fig. $195 \%$ confidence ellipsoids of the two reference examples.

We studied the influence of different parameter setting on the accuracy of the estimation. First we note that we may chose $\bar{\lambda}=1$ for simplicity, since the estimation of this parameter is independent from that of the others. A major characteristic of a Hawkes process is its the integral of the IRF, denoted by $c$, see (3), defining different regimes w.r.t. stability. A second feature that we considered is simply the attenuation determined by the parameter $a$.

We simulated numerous Hawkes processes ( 9 regimes at 10 different $a$-s, $N=$ $10.000)$, and computed the volume of the confidence ellipsoids $\left(V_{C E}\right)$. The dependence of $V_{C E}$ on the particular regime $c$ and the attenuation $a$ is demonstrated on Figure 2. On the left hand side it is seen that $V_{C E}$ is a monotone decreasing function of $a$ for each regime. On the right hand side $V_{C E}$ is depicted as a function of $c$ for various choices of $a$. It is interesting to observe that estimation problem becomes more difficult for values of $c$ close to 1 or 0 .
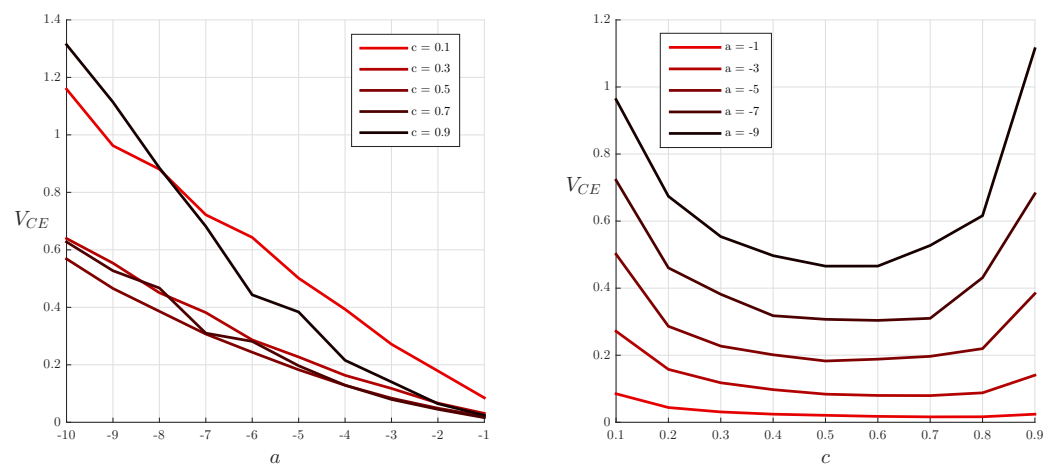

Fig. 2 Left: $V_{C E}$ vs. $a$. Right: $V_{C E}$ vs. $c$ 
In order to understand the details about the increased uncertainty of the estimators when $c$ is close to 1 or 0 , we compute the asymptotic standard deviation (ASD) of individual parameters, which are simply the diagonal elements of $I^{-1}\left(\theta^{*}\right)$. To make different parameter-settings comparable we normalized these values by $V_{C E}^{1 / 3}$, see Figure 3. These results show that the overall uncertainty, when $c$ is close to 1 or 0 , is due to the uncertainty in the estimation of $\alpha$ for $c$ close 0 , and that of $\bar{\lambda}$ for $c$ close 1 . The accuracy of the estimation of $\sigma$ is quite satisfactory for all values of $c$.
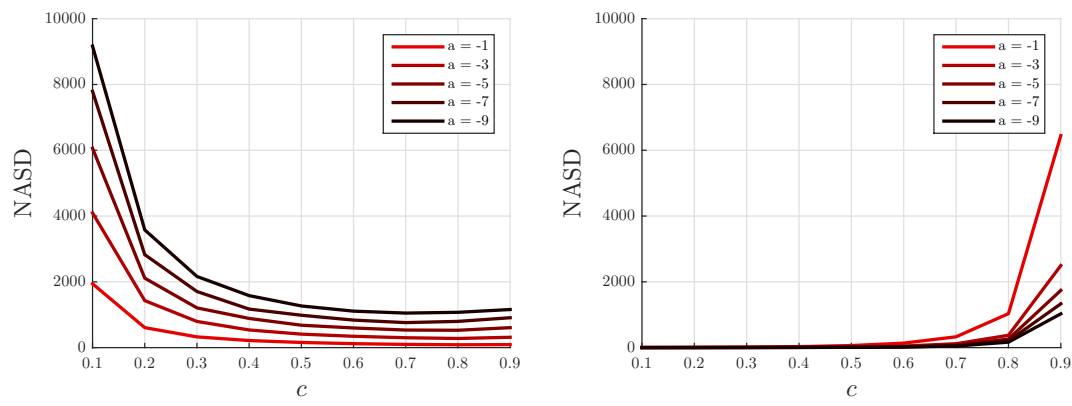

Fig. 3 Normalized asymptotic standard deviation (NASD) of $\alpha$ and $\bar{\lambda}$, respectively.

The shift in the degree of uncertainty between $\alpha$ and $\bar{\lambda}$ indicates a change in the orientation of the confidence ellipsoid. This finding may be used to detect regimechanges more efficiently, and ultimately to detect changes in the brain-state.

\section{Discussion}

Forecasting seizures with application in closed-loop neurostimulators is of great need for patients with therapy-resistant epilepsy. With the expanding arsenal of clinical neurophysiology it is becoming possible to monitor patients' brain-activity at a cellular level [16]. Therefore seizure prediction based on information extracted from neuronal firing is a promising research topic.

A convenient framework to describe neuronal firing patterns in a compressed manner are self-exiting point processes or Hawkes processes. When fitting Hawkes processes to simulated or real-world data critical factors are both the computability and the statistical reliability of the MLE. In the present experimental mathematical research we explored the sensitivity of the MLE method for the class Hawkes processes with exponential IRF for a large variety of models. The models were classified via a single parameter related to stability, defining different regimes. We found that the estimation accuracy of parameters (pattern of uncertainty) highly depends on the actual regime. 
The ultimate measure of accuracy is the applicability of the methods to discriminate between different brain-states based on experimental data. A further step towards real life applications is the integration of our experimental findings for the off-line MLE method into the development of a reliable on-line MLE method along the lines proposed back in [5], to be discussed in a forthcoming paper.

Acknowledgements This research has been partially supported by the European Union, cofinanced by the European Social Fund (EFOP-3.6.3-VEKOP- 16-2017-00002).

\section{References}

1. Brémaud, P.: Point Processes and Queues: Martingale Dynamics, Springer Verlag, New York (1981)

2. Chornoboy, E.S., Schramm, L.P., Karr, A.F.: Maximum likelihood identification of neural point process systems. Biol. Cybern. 59: 265-75, 1988.

3. Daley, D.J., Vere-Jones, D.: An Introduction to the Theory of Point Processes. Springer Science \& Business Media, New York (2013)

4. Gadhoumi, K., Lina, J-M., Mormann, F., Gotman, J.: Seizure prediction for therapeutic devices: a review. J. Neurosci. Methods $029: 1-13,2015$.

5. Gerencsér, L., Matias, C., Vágó, Z., Torma, B., Weiss, B.: Self-exciting point processes with applications in finance and medicine. In: Proc. of the 18th International Symposium on Mathematical Theory of Networks and Systems (MTNS2008), Virginia Tech, Blacksburg, Virginia, USA, 2008.

6. Gerhard, F., Deger, M., Truccolo, W.: On the stability and dynamics of stochastic spiking neuron models: Nonlinear Hawkes process and point process GLMs. PLoS Comp. Biol. 13: e1005390, 2017, https://doi.org/10.1371/journal.pcbi.1005390

7. Hansen, N.R., Reynaud-Bouret, P., Rivoirard, V.: Lasso and probabilistic inequalities for multivariate point processes. Bernoulli 21: 83-143, 2015.

8. Hawkes, A.G.: Spectra of some self-exiciting and mutually exciting point processes. Biometrika 58: 83-90, 1971.

9. Kuhlmann, L., Grayden, D.B., Wendling, F., Schiff, S.J.: Role of multiple-scale modeling of epilepsy in seizure forecasting. J. Clin. Neurophysiol. 32: 220-226, 2015.

10. Merricks, E.M., Smith, E.H., McKhann, G.M., Goodman, R.R., Bateman, L.M., Emerson, R.G., Schevon, C.A., Trevelyan, A.J.: Single unit action potentials in humans and the effect of seizure activity. Brain 138: 2891-2906, 2015.

11. Møller, J., Rasmussen, J.G..: Perfect simulation of Hawkes processes. Adv. Appl. Probab. 37: 629-646, 2005.

12. Ozaki, T.: Maximum likelihood estimation of Hawkes' self-exciting point processes. Ann. Inst. Stat. Math. 31: 145-155, 1979.

13. Quiroga, R.Q., Nadasdy, Z., Ben-Shaul, Y.: Unsupervised spike detection and sorting with wavelets and superparamagnetic clustering. Neural. Comput. 16: 1661-1687, 2004.

14. Schulze-Bonhage, A., Kühn, A.: Unpredictability of seizures and the burden of epilepsy. In: Seizure Prediction in Epilepsy: From Basic Mechanisms to Clinical Applications. 2008, p. $1-10$.

15. Truccolo, W., Donoghue, J.A., Hochberg, L.R., Eskandar, E.N., Madsen, J.R., Anderson, W.S., Brown, E.N., Halgren, E., Cash, S.S.: Single-neuron dynamics in human focal epilepsy. Nat. Neurosci. 14: 635-641, 2011.

16. Ulbert, I., Maglóczky, Z., Erôss, L., Czirják, S., Vajda, J., Bognár, L., Tóth, S., Szabó, Z., Halász, P., Fabó, D., Halgren, E., Freund, T.F., Karmos, G.: In vivo laminar electrophysiology co-registered with histology in the hippocampus of patients with temporal lobe epilepsy. Exp. Neurol. 187: 310-318, 2004. 